\title{
La desigualdad socioterritorial y el bienestar económico: Una propuesta metodológica
}

\section{Socio-territorial inequality and economic well-being: A methodological proposal}

\author{
Luis Tinedo ${ }^{1}$ (iD $*$
}

\section{RESUMEN}

Este artículo científico detalla el análisis de la desigualdad desde una perspectiva socioterritorial e integra los principales paradigmas que limitan el bienestar económico de las regiones, no obstante, el propósito es construir un esquema metodológico que relaciona las dimensiones de las desigualdades socioterritoriales y el bienestar económico. Para ello, se presenta un análisis inductivo deductivo que involucra la técnica documental y observacional. De esta forma se identifica claramente que la pobreza y la vulnerabilidad tienen una relación estrecha con la desigualdad social y territorial, de ello se rescata que las dimensiones como el espacio geográfico, las condiciones de la vivienda, las características del hogar, la participación ciudadana y la tecnología son esenciales para catalogar la disparidad existente en las regiones pobres y vulnerables; así mismo, se muestra que el bienestar económico depende del nivel de empleo, participación productiva, nivel de ingreso y nivel de gasto en consumo. Para concluir, la investigación es un eslabón para generar interés por parte del Estado en la formulación de políticas gubernamentales basados en la realidad de las comunidades, descubriendo sus ventajas comparativas que surgen a raíz de la necesidad de tener una mejor calidad de vida.

Palabras clave: Desigualdad socioterritorial, bienestar económico, política gubernamental.

\begin{abstract}
This scientific article details the analysis of inequality from a socio-territorial perspective and integrates the main paradigms that limit the economic well-being of the regions; however, the purpose is to build a methodological scheme that relates the dimensions of socio-territorial inequalities and economic wellbeing. For this, an inductive deductive analysis is presented that involves the documentary and observational technique. In this way it is clearly identified that poverty and vulnerability have a close relationship with social and territorial inequality, from this it is rescued that dimensions such as geographical space, housing conditions, household characteristics, citizen participation and technology is essential to catalog the existing disparity in poor and vulnerable regions; Likewise, it is shown that economic well-being depends on the level of employment, productive participation, level of income and level of consumption expenditure. To conclude, the research is a link to generate interest on the part of the State in the formulation of government policies based on the reality of the communities, discovering their comparative advantages that arise as a result of the need to have a better quality of life.
\end{abstract}

Keywords: socio-territorial inequality, economic well-being, government policy.

DOI: https://doi.org/10.37787/pakamuros-unj.v8i3.140

Recibido: 04/10/2020. Aceptado: 28/10/2020

* Autor para correspondencia 


\section{INTRODUCCIÓN}

La realidad de muchos países latinoamericanos es muy compleja y diversa, como resultado de los problemas latentes de origen económico, social, territorial, ambiental y cultural, de hecho, en los primeros 15 años del siglo XXI la Organización de las Naciones Unidas (ONU) a través de la reunión de sus líderes mundiales acordaron implementar 17 objetivos de desarrollo sostenible enlazados a cada prioridad de política pública; dentro de ese contexto aparece el objetivo diez denominado reducción de las desigualdades (ONU, 2015) de hecho, el tema aterriza en la lucha denodada para reducir la pobreza. Dentro de la problemática surge una ventana para el análisis y desarrollo de la desigualdad socioterritorial como limitante directo del bienestar económico de los hogares de cada región de un Estado. Las dificultades surgen a partir de la heterogeneidad territorial, donde se muestra la cohesión dispar de la distribución de la riqueza y la calidad de vida material, señalando que existen muchos territorios estancados con un alto grado de pobreza, en adición a lo anterior, se puede afirmar que el territorio sería un eje causal para elucidar las desigualdades sociales (Cepal, 2016).

Las desigualdades perjudican el bienestar económico de las familias y limitan la calidad de vida, en dicho contexto, Cuervo y Morales (2008) descubrieron que las desigualdades se encuentran explicadas en el comportamiento de variables sensibles como el nivel de vida y el bienestar de las comunidades que constituyen un territorio, involucrado estrechamente con el estudio del desarrollo local. De esta manera, en algunas regiones con mucha polaridad y desigualdad existe cohesión social lo que conlleva a una creciente inestabilidad económica y productiva (Wong, 2001), en reiteradas ocasiones las desigualdades pueden ser un resultado de interrelaciones económicas, que existen entre los agentes económicos (familias, empresas y Estado). Así mismo, según Canelos (2018) con el propósito de analizar el nivel de desigualdad en distintas ciudades del Ecuador, determinó que el problema de la desigualdad es estructural debido a la heterogeneidad de sus dimensiones (espacial, económica, social productiva e institucional), en ella se reconocen cinco factores decisivos que son la concentración poblacional, las disparidades de la estructura productiva dentro y fuera de los sectores, la composición empresarial, la población económicamente activa y las diferencias en las condiciones de vida de las familias, además, Fernández, Nora, y Hinojosa (2016) aterrizaron en un análisis más detallado, y determinaron que la desigualdad socio regional es el resultado de los procesos históricos de acumulación y des acumulación de capital, en tal sentido, es dinámica y se debe a los procesos de recursos humanos, económicos, políticos y sociales que inciden en las relaciones intersectoriales. Los hechos ineludibles configuran que la desigualdad social 
territorial es un problema de alta complejidad, siendo, un limitante para lograr el bienestar pleno de las comunidades.

La desigualdad socioterritorial se puede contextualizar como un paradigma complejo porque se evidencia una diferenciación muy holgada en las condiciones educativas, hogares, servicios básicos y estructura agrícola que limitan el crecimiento y bienestar económico de muchas poblaciones ubicadas en distintas áreas geográficas (Krapovickas y Garay, 2017), cabe señalar que Czytajlo (2017), describe a dicho concepto como la fragmentación que se expresa en indicadores socioeconómicos y en el acceso real de la población a las ofertas urbanas. Sin embargo, no hay que olvidar los orígenes de la teoría planteada por Simon Kuztnets en 1955. En dicho estudio se evidenció que el crecimiento económico fomentado por el sistema capitalista es causante de la desigualdad en el ingreso, planteándose de esta manera, la muy reconocida curva de Kuztnets (Kuznets, 1955), de hecho, el problema de los procesos socioeconómicos surgen a partir del análisis de los sectores industrial y agrícola, calando en la caracterización de la población urbana y rural (Modrego y Cazzuffi , 2015). Por otro lado, el bienestar económico no solo se limita al estudio de la renta per cápita, sino, va más allá, donde encuadra un criterio multidimensional relacionado al desarrollo humano, calidad educativa, calidad de salud, rasgos sociales e incluso características ambientales (Murias, Martínez, y Novello, 2009). La clave del aspecto teórico radica en la investigación de Nordhaus y Tobin (1973), donde plantean un método para medir el bienestar, cabe resaltar que la variable estudiada fue el gasto del consumo individual. Sin embargo, la mejor propuesta teórica se alinea con la expuesta por Osberg y Sharpe (2003) donde el bienestar económico depende de los flujos de consumo per cápita efectivo actual; acumulación social neta de existencias de recursos productivos; la distribución del ingreso; y seguridad económica.

Consolidando una nueva perspectiva, se realiza un diagnóstico critico que pretende profundizar las caracterizaciones socioterritoriales que limitan en parte el bienestar económico; a menudo, existen asimetrías negativas por causa de la desigualdad que se evidencian en las poblaciones ubicadas en diferentes áreas geográficas de un país. Cabe destacar, que la problemática desarrollada se basa en el planteamiento de la siguiente pregunta ¿Las desigualdades socioterritoriales son causales del estancamiento económico de las familias?, de esta forma, para responder a dicha interrogante se desarrollará una construcción metodológica critica de las desigualdades socioterritoriales en un ámbito del bienestar económico y desarrollo local. 


\section{MATERIALES Y MÉTODOS}

La investigación se enfoca en el análisis documental que involucran las variables: la desigualdad socioterritoral y bienestar económico, no obstante, tiene un alcance descriptivo por que examina la teoría científica estudiada para definir conceptos que permiten desarrollar una construcción metodológica de las variables en estudio. Además, se usó el método inductivo deductivo para determinar una conclusión general basado en la observación de hechos, no obstante, las técnicas de recolección de datos utilizados fue la observación directa y la documentación bibliográfica, cabe mencionar que los instrumentos o fuentes de datos se obtuvo de las fichas bibliográficas y análisis documental.

La estrategia de la metodología consistió en la combinación de las técnicas de investigación cualitativa constructivista, usando las herramientas principales de observación y análisis documental para visualizar el contexto constructivo de la desigualdad socioterritorial como limitante del bienestar económico. En realidad, se debe mencionar que la mayoría de información obtenida para este artículo fue secundaria, es por ello, que a partir de la premisa se realizó una construcción metodológica que remarquen las dimensiones paradigmáticas de las variables.

En concordancia a lo mencionado la investigación se llevó a cabo mediante el siguiente procedimiento:

- Se revisó material bibliográfico sobre el tema de investigación.

- Se analizó los antecedentes y las teorías donde se sustenta el artículo.

- Se presentan los resultados a partir de la construcción del esquema metodológico donde se enlazan las variables de estudio.

- Se elaboró la discusión contrastando los resultados con la literatura desarrollada.

- Se elaboró las conclusiones

En consecuencia, el diseño es no experimental porque no se hizo manipulación de las variables, así mismo la investigación es de corte transversal de alcance descriptivo.

\section{RESULTADOS}

Los estudios modernos, basados en la experticia de las teóricas antiguas, tratan de explicar lo que muchos países catalogan desigualdad, como una problemática compleja, además, los seres racionales sabemos que el mundo está constituido por muchos Estados independientes, desde el origen de la humanidad hemos aprendido a convivir, conjugando procesos que nos permitieron identificar problemas latentes en las sociedades diversas. Para una mejor percepción, durante el transcurrir del tiempo, se ha lidiado con los procesos económicos, sociales, territoriales, ambientales y culturales, causales del malestar de la civilización actual, el mundo como se ve ya no es el mismo. Ahora bien, la Organización de las Naciones 
Unidas (ONU), con un interés particular puso en la mesa la agenda 2030 con la propuesta de 17 objetivos de desarrollo sostenible, con el único fin erradicar la pobreza; dentro de ello la desigualdad forma parte de una lista interminable de factores que inciden en el estancamiento y limitan el bienestar de la población. Esto nos lleva a meditar en un entorno de incertidumbre y nos preguntamos: ¿Cómo reducir la desigualdad?; la pregunta es simple, sin embargo, muy compleja de responder. Cabe señalar que el problema es de constante preocupación en el mundo, más significancia toma en los países de esta orbita territorial, Latinoamérica.

Por otro lado, la sociedad es un sistema complejo, que busca establecerse en un ámbito difícil de dominar, debido a las constantes transiciones históricas, por ello el territorio es un factor preponderante bajo una perspectiva racional. Desde este aspecto las relaciones que se presentan entre el territorio, la sociedad y el bienestar son de carácter estructural y no coyuntural, presentándose secciones relevantes de carácter, geográfico, generacional, racial, étnico, divergencia social, lingüístico y tecnológico, de tal forma, todo ello lucida rasgos concluyentes para converger en desigualdades socioterritoriales. Esto permite reconocer brechas, que son el punto de partida para realizar diagnósticos específicos, no olvidar, que la perspectiva positiva para identificar oportunidades de crecimiento permite generar ámbitos de confort para acceder a los derechos fundamentales. Se puede decir que los territorios se estancan por la masiva migración del campo a la ciudad, por los escases de oportunidades, o la era tecnológica; siempre las zonas urbanas frente a las zonas rurales tienen grandes diferencias, sin embargo, la política gubernamental tampoco ayuda en un sentido común para crear integración desde un punto de vista comunal. A grueso modo desde una tendencia positivista se pretende construir una propuesta metodológica para representar las desigualdades socioterritoriales, que involucra barreras y dimensiones limitantes del desarrollo y bienestar económico.

El planteamiento de una propuesta metodológica, basada en la observación y el análisis documental, hará eco en áreas geográficas y regiones poco desarrolladas. Los análisis presentados por Fernández, Nora, y Hinojosa (2016); Czytajlo (2017); Cuervo y Morales (2008); y Cepal (2016) determinan que la desigualdad es dimensional, sin embargo, existe un vacío estrecho para definir una tendencia o metodología que ayude a explicar el comportamiento de la disparidad socioterritorial en países como el Perú. A mérito de ello en la Figura 1 se presenta un esquema sintético que aborda las desigualdades socioterritoriales contextualizadas desde un punto de vista paradigmático. 


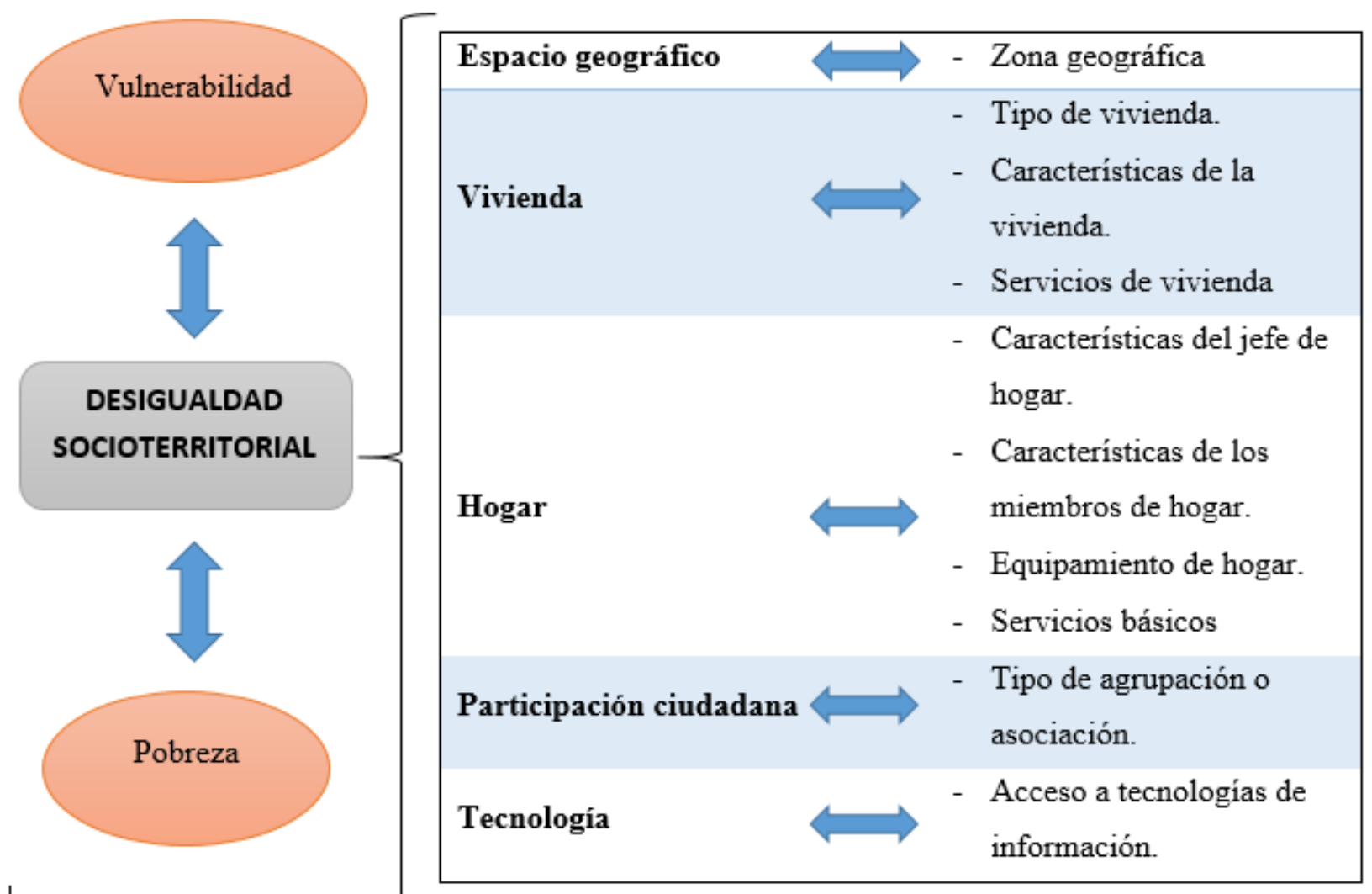

Figura 1. Esquema representativo de la desigualdad socioterritorial; Elaboración propia basado en Fernández, Nora, y Hinojosa (2016); Czytajlo (2017); Cuervo y Morales (2008) y Cepal (2016).

La Figura 1, representa una construcción esquemática de las desigualdades socioterritoriales, en ella aparecen cinco dimensiones muy remarcadas, la ubicación geográfica, vivienda, hogar, participación ciudadana y la tecnología. Destacando la problemática de las zonas rurales y urbanas, se elaboró esta metodología, a diferencia de otras construcciones, la dimensión tecnológica hoy en día es una barrera imponente que crea desigualdad social y territorial por que limita a muchas familias la realización de actividades importantes, a menudo, la dificultad es permanente por la reducida inversión pública, el mercado imperfecto de las telecomunicaciones y la escasez de capacidad de gasto, son detonantes que impiden frenar la brecha digital en regiones pobres y extremadamente pobres. En el contexto, se resalta que la desigualdad socioterritorial tiene una relación estrecha con la pobreza y la vulnerabilidad, dicha afirmación se puede corroborar con la observación de vivencias cotidianas enlazadas con las costumbres e interrelaciones comunales propias de cada sociedad. 
Una población gozará de bienestar cuando cubran sus necesidades de forma satisfactoria, es decir, tienen que llegar a la próspera calidad de vida; coincidiendo con lo ya expuesto por Murias, Martínez, y Novello (2009), el ingreso no es el factor preponderante para alcanzar dicho bienestar, también, persiste la salud, educación, participación productiva, empleo y otros factores recurrentes, que forman la piedra angular para lograr el crecimiento y desarrollo de los países. Desde un punto de vista macroeconómico Nordhaus y Tobin (1973); Osberg y Sharpe (2003); ligados en el análisis de Murias, Martínez, y Novello (2009), el bienestar económico es multidimensional, concordando plenamente con dichos investigadores; no obstante, la caracterización del pueblo peruano y latinoamericano es particular por factores relacionados al medio ambiente, historia, lengua y cultura. Complementando a lo analizado líneas arriba, se exhibe la Figura 2, como resultado de una exploración documental detallada que explica el comportamiento del bienestar económico a través de la inserción de sus principales dimensiones básicas.

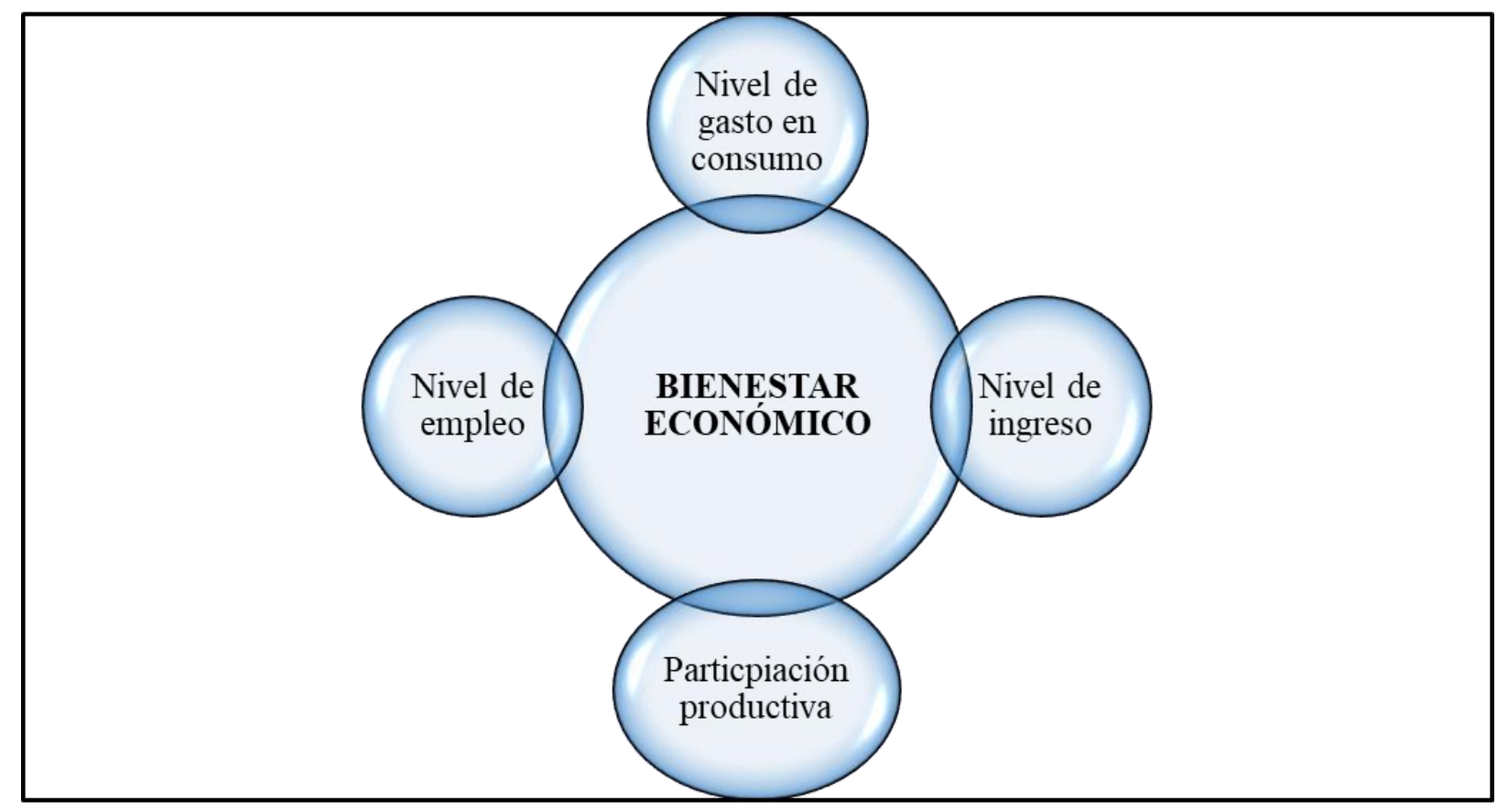

Figura 2. Esquema multidimensional del bienestar económico; Fuente: Elaboración propia basado en Murias, Martínez, y Novello (2009); Nordhaus y Tobin (1973); Osberg y Sharpe (2003).

Las caracterizaciones que se muestran en la Figura 2, podrían ser determinantes valorativos para generar bienestar económico, la búsqueda de este paradigma permuta con la reducción de las desigualdades en 
los territorios diferenciados. En contraste con lo convencional, se presenta cuatro dimensiones primordiales para explicar la naturaleza del comportamiento del bienestar desde el punto de vista económico. Consecuentemente, las familias con escasos recursos, tienen que enfrentar los problemas cotidianos de las regiones que involucra un gasto familiar ajustado, alto nivel de desempleo, escasa participación productiva e ingreso mínimo; insertado a estos hechos las cuestiones coyunturales de política interna. Todo ello forma un círculo vicioso que genera pobreza. Por otra parte, la cosmovisión sociocultural enfrentada con la modernidad puede ser un limitante para generar bienestar económico.

Las constantes luchas por reducir la pobreza, en este siglo XXI, conlleva a generar un hito de transformación, por ello la diferenciación de los espacios geográficos, la precariedad de las condiciones de vivienda y hogar, la indiferencia entre la ciudadanía y la brecha digital determinan la persistencia de la desigualdad en el territorio y la sociedad, especialmente en regiones pobres. Por consiguiente, las acciones no articuladas generadas por el Estado muestran indicios de debilidad; es así, la reducción del ingreso, el contante desempleo, el gasto de consumo mínimo y la escasa participación productiva, favorecen la permanencia de problemas suscitados en países subdesarrollados; cabe mencionar, que los factores expuestos son condicionantes de la calidad de vida de las personas, por consiguiente, influyen en el bienestar económico. En resumen, la conexión de las dimensiones entre la desigualdad socioterritorial y el bienestar económico forman un eje angular para generar acciones conjuntas que involucran a los agentes económicos en el logro de un objetivo común (Figura 3).

\begin{tabular}{|c|c|c|c|}
\hline \multicolumn{4}{|c|}{ DESIGUALDAD SOCIOTERRITORIAL } \\
\hline $\begin{array}{l}\text { Espacio } \\
\text { Geográfico }\end{array}$ & Vivienda & $\begin{array}{c}\text { Participación } \\
\text { Ciudadana }\end{array}$ & Tecnología \\
\hline \multicolumn{4}{|c|}{ BIENESTAR ECONÓMICO } \\
\hline $\begin{array}{l}\text { Nivel de gasto en } \\
\text { consumo }\end{array}$ & Nivel de ingreso & empleo & $\begin{array}{l}\text { Participación } \\
\text { ciudadana }\end{array}$ \\
\hline
\end{tabular}

Figura 3. Relación causal entre la desigualdad socioterritorial y bienestar económico; Fuente: Elaboración propia basado en Fernández, Nora, y Hinojosa (2016); Czytajlo (2017); Cuervo y Morales (2008); Cepal (2016); Murias, Martínez, y Novello (2009); Nordhaus y Tobin (1973); Osberg y Sharpe (2003)

Consecuentemente, los ciudadanos debemos de incentivar las transformaciones socioterritoriales, ubicarse en el tramo favorable para generar incentivos en las familias vulnerables, de esta forma, tomar como ejemplo, países del primer mundo que experimentaron procesos de cambios generacionales, 
coyunturales y estructurales, a base de la inversión prioritaria en sectores estratégicos, como la educación, que produce conocimiento e incentiva el talento y la innovación; además, la fragmentación de la sociedad y el territorio por causa del sistema capitalista, ahonda en la necesidad de querer progresar, aunque el trabajo es denodado por que las brechas de territorio son amplias en áreas locales, sin embargo, la población conjuntamente con el Estado deben tomar la concepción de desarrollo local para implementar política gubernamental a partir de las necesidades directas de las comunidades pluriculturales.

\section{DISCUSIÓN}

El remarcado debate sobre la desigualdad socio territorial se estructura en un punto de quiebre denominado heterogeneidad de los territorios, (Cepal, 2016, pág. 61 ) manifiesta que existe una holgada brecha entre zonas geográficas pobres y ricas, de esta manera concuerdo en que una de las dimensiones referentes para medir la variable estudiada es la ubicación geográfica, a menudo las desigualdades son limitantes de la calidad de vida y por ello se dimensionan teniendo en consideración muchos paradigmas como la estabilidad económica y el bienestar de las comunidades locales, concordando con lo expuesto por (Cuervo y Morales, 2008; Wong, 2001), ya que dicho problema surge por fallas de las interrelaciones economías entre los agentes. La propuesta paradigmática de la desigualdad socioterritorial presentada emula cinco dimensiones remarcadas (espacio geográfico, vivienda, hogar participación ciudadana y tecnología) en concordancia con lo expuesto por (Fernández, Nora, y Hinojosa (2016); Czytajlo (2017); Cuervo y Morales (2008)). Sin embargo, las dimensiones propuestas por Krapovickas y Garay (2017), manifestando que las limitantes del bienestar se relacionan a las condiciones educativas, hogares, servicios básicos y estructura agrícola, coincidiendo en la propuesta metodológica que se presenta en los resultados (Figura 1).

Según la perspectiva dimensional el bienestar económico no solo depende del ingreso sino de otras dimensiones como el empleo, el gasto de consumo y la participación productiva en concordancia con lo estipulado por (Murias, Martínez, y Novello (2009); Nordhaus y Tobin (1973); Osberg y Sharpe (2003)), no obstante, (Modrego y Cazzuffi , 2015) basado en el estudio de (Kuznets, 1955), determina que la desigualdad limita el crecimiento económico en las zonas rurales y urbanas, de esta forma, podemos decir que efectivamente la brechas son amplias en diversos sectores de la economía y ello impide articular la política gubernamental desde un punto de vista del desarrollo local. 


\section{CONCLUSIONES}

El estudio evidencia que las desigualdades perjudican el bienestar de las familias, colocando barreras muy fuertes que limitan el crecimiento y desarrollo de los países, bajo este contexto, la desigualdad socioterritorial de acuerdo a los estudios revisados, es un problema complejo que involucra dimensiones muy remarcadas en los espacios, a menudo, los grupos sociales ubicados en territorios estancados pueden integrarse a los desarrollados a través de una política gubernamental articulada. El ritmo de crecimiento de muchas sociedades depende las relaciones socioeconómicas y los espacios territoriales, sin embargo, se evidencia una marcada desarticulación entre los planes de gobierno y la realidad local. Otro elemento que alimenta la desigualdad es la vulnerabilidad, conjuntamente con la divergencia entre la distribución del ingreso y la institucionalidad débil, generan negatividad en los propósitos planificados de las sociedades, esto detona en el fracaso de los países del tercer mundo. La posibilidad de fortalecer las instituciones es posible, pero, se debe aterrizar en propuestas que fortalezcan la justicia e inclusión social. De esta manera, los estados están en la constante búsqueda de estrategias y políticas que contribuyan con el bienestar de las familias. Recabando y discutiendo los estudios realizados se presenta una construcción esquemática para anidar las desigualdades socioeconómicas y el bienestar económico.

Los esquemas propuestos (Figura 1 y Figura 2), basados en la investigación documental, permiten sintetizar el estudio profundo de las desigualdades socioterritoriales y el bienestar económico, esto cabe mencionar basado en el análisis multidimensional. La metodología constructiva muestra que la pobreza y la vulnerabilidad tienen una relación estrecha con la desigualdad social y territorial, consistentemente, la dimensiones como el espacio geográfico, las condiciones de la vivienda, las características del hogar, la participación ciudadana y la tecnología son eslabones clave para caracterizar la disparidad existente en las regiones pobres y vulnerables (Figura 1), Así mismo, las dimensiones propuestas para explicar el bienestar económico son el nivel de gasto, nivel de empleo, participación productiva y el nivel de ingreso (Figura 2). En relación a lo mencionado, los esquemas aclaran una idea perceptiva para luchar contra la pobreza, basado en la concepción del desarrollo local y la política gubernamental, sin dejar de lado a los actores primarios que son las comunidades pluriculturales. Simultáneamente, la Figura 3 enlaza una conexión estrecha de causa efecto entre la desigualdad socioterritorial y el bienestar económico, aclarando, que dicho problema es una fuerte barrera que impide lograr una mejor calidad de vida en las familias.

En síntesis, la investigación es un punto de partida, para generar interés por parte del Estado en la formulación de políticas basados en la realidad de las comunidades, descubriendo sus ventajas 
comparativas que surgen a raíz de la necesidad de tener una mejor calidad de vida. En relación a lo anterior, hay mucho por recorrer en un ámbito investigativo que involucra, desigualdad, vulnerabilidad, pobreza, desarrollo, crecimiento, territorio y sociedad.

\section{REFERENCIAS BIBLIOGRÁFICAS}

Canelos, R. (2018). La Desigualdad espacial en Ecuador: un enfoque de brechas estructurales (20022017). Quito: Universidad Autonoma de Barcelona. Obtenido de https://ddd.uab.cat/pub/tesis/2018/hdl_10803_666602/rcs1de1.pdf

Cepal. (2016). La matriz de la desigualdad social en América Latina. Santigo de Chile: Naciones Unidas. Obtenido de https://repositorio.cepal.org/bitstream/handle/11362/40668/S1600946_es.pdf?sequence=4\&isAl lowed=y

Cuervo, M., \& Morales, F. (2008). Las teorías del desarrollo y las desigualdades regionales. Analisis Economico, 365-383.

Czytajlo, N. P. (2017). Desigualdades socio-territoriales y de género en espacios metropolitanos: El Sistema metropolitano de Tucumán (2001-2010). Bitacora 27(3), 121-134. Recuperado el 16 de Setiembre de 2020, de https://doi.org/10.15446/bitacora.v27n3.66484

Fernandez , H., Nora, E., \& Hinojosa, C. (2016). Desigualdad Socioregional y Politicas Públicas en Tamaulipas. SOCIOTAM, XXVI(1), 191-218. Recuperado el 14 de Setiembre de 2020, de https://www.redalyc.org/articulo.oa?id=65456116009

Krapovickas, J., \& Garay, A. (2017). Una aproximación descriptiva a la desigualdad socioterritorial en ambitos rurales del Noroeste Argentino en la primera decada del siglo XXI. Estudios Geograficos, 605-632.

Kuznets, S. (1955). Economic Growth and Income Inequality. The American Economic Review, 45(1), 1-28. Recuperado el 19 de Setiembre de 2020, de http://courses.nus.edu.sg/course/ecshua/eca5374/Economics\%20growth\%20and\%20income\%2 Oinequality_Kuznets_AER55.pdf

Modrego , F., \& Cazzuffi , C. (2015). Desigualdad y crecimiento económico: contribuciones desde el desarrollo territorial. Santiago de Chile: Centro Latnoamericano para el Desarrollo Rural. Obtenido de http://www.rimisp.org/wp- 
content/files_mf/1434663450155CCazzuffi2015_04_24_Desigualdadycrecimiento_vfinal_edita do.pdf

Murias, P., Martínez, F., \& Novello, S. (2009). Bienestar económico regional: un enfoque comparativo entre regiones españolas e italianas. Investigaciones Regionales(18), 5-36.

Nordhaus, W., \& Tobin, J. (1973). Is Growth Obsolete? (M. Moss, Ed.) The Measurement of Economic and Social Performance, 509-564. Recuperado el 19 de Setiembre de 2020, de https://www.nber.org/chapters/c3621.pdf

ONU . (25 de Setiembre de 2015). Objetivos de Desarrollo Sostenible. Obtenido de Orgnanización de las Naciones Unidas : https://www.un.org/sustainabledevelopment/es/objetivos-de-desarrollosostenible/

Osberg, L., \& Sharpe, A. (2003). Un índice de bienestar económico para países seleccionados de la OCDE. La biblioteca electrónica de SSRN, 291-316. Obtenido de https://papers.ssrn.com/sol3/papers.cfm?abstract_id=325161

Wong, P. (2001). Desigualdades regionales y cohesión social: viejos dilemas y nuevos desafíos. México: Mauricio de María y Campos y Georgina Sánchez, editores. 